\title{
Characteristic alterations of long-loop "reflexes" in patients with Friedreich's disease and late atrophy of the cerebellar anterior lobe
}

\author{
HC DIENER, J DICHGANS, M BACHER, B GUSCHLBAUER \\ From the Neurologische Universitätsklinik, Tübingen, Federal Republic of Germany
}

SUMMARY Sudden tilting of a platform around the axis of the human ankle joint causes a regular pattern of short and medium latency EMG responses in the stretched triceps surae muscle and a long latency response in its antagonist, the anterior tibial muscle. This paper reports alterations of these EMG responses in 41 patients with cerebellar lesions. Patients with anterior lobe atrophy exhibited normal latencies of all EMG responses, but an increase in duration and amplitude of the long latency response. This may at least partially explain the $3 \mathrm{~Hz}$ postural tremor, which can be evoked in these patients through a toe-up tilt of the platform. Patients with lesions restricted to the vestibulocerebellum and to the cerebellar hemispheres exhibited normal latencies of all EMG responses. The most specific finding in all of the patients suffering from Friedreich's ataxia (affecting spinal afferents) was the massive delay of the stabilising response of the anterior tibial muscle. The fact that the patients with lesions restricted to the cerebellum invariably exhibited normal latencies of postural "reflexes" indicates that the exact "timing" of these responses is independent of the cerebellum. The duration and amplitude of the long latency response of the antagonist, however, seems to be controlled by the cerebellum.

Sudden tilts of the support on which a subject stands causes a regular pattern of EMG responses in the stretched leg muscles and their antagonists. ${ }^{1-3}$ The early EMG response in the stretched triceps surae muscle with a latency of about $50 \mathrm{~ms}$ (short latency) corresponds to a segmental stretch reflex..$^{4-8}$ The nature of the subsequent medium latency response in the stretched muscle (latency $105 \mathrm{~ms}$ ) is not clear. This EMG response corresponds to the "long loop response" observed in human arm muscles ${ }^{9-11}$ and leg muscles. ${ }^{78^{12-14}}$ The question whether medium latency travels via a transcortical loop ${ }^{15}$ or exclusively via polysynaptic spinal pathways ${ }^{16} 17$ and/or is due to segmented afferent bursts of spindle activity ${ }^{18}$ cannot be answered at the moment. Early and medium latency responses are functionally destabilising posture under our experimental conditions, since they enforce the induced passive backward displacement of the body. Stabilisation of upright posture is mainly achieved by EMG

Address for reprint requests: Dr HC Diener, Neurologische Universitätsklinik, Liebermeisterstr 18-20, D-7400 Tübingen, FRG.

Received 26 July 1983 and in revised form 12 December 1983. Accepted 22 December 1983 responses of long latency $(130 \mathrm{~ms})$ in the antagonist of the stretched muscle. ${ }^{19}$ This late EMG response restores the initial body position and is not voluntarily performed. ${ }^{3}$ It exhibits some analogies with the M3-response in arm muscles.'

This paper reports experiments in patients with diseases of the cerebellum and spino-cerebellar degenerations. These were undertaken in an attempt to contribute to the still open question of whether or not the cerebellum is involved in the generation of medium and long latency responses to muscle stretch or at least the modulation of their latency, amplitude or duration. Of particular interest are patients with late atrophy of the anterior lobe of the cerebellum due to alcoholism ${ }^{20-22}$ since an earlier study reports enhanced and delayed long loop reflexes which were supposed to cause the typical $3 \mathrm{~Hz}$ postural tremor of these patients. ${ }^{23}$

\section{Methods}

Subjects stood on a force measuring platform, which could be rotated around the axis of the ankle joint toe-up or toe-down with a velocity of $50 \mathrm{o} / \mathrm{s}$ and an amplitude of $4^{\circ}$. The displacement of the centre of foot pressure (CFP) was 
computed from the forces acting on the strain gauges at the four corners of the platform. Angular displacements of the head and hip were recorded by means of goniometers laterally attached to belts around the head and hip. EMGs of the anterior tibial (TA) and triceps surae (TS) muscles were recorded with surface electrodes. EMG signals were preamplified, full wave rectified and band-pass filtered (1.6 $\mathrm{Hz}-1 \mathrm{kHz}$ ). The displacements of the CFP, head and hip were averaged over eight corresponding runs (Nicolet 1070 ) and displayed on an $x-y$ plotter. The time interval between single platform tilts varied randomly between 5 and $12 \mathrm{~s}$. EMG latencies were measured in terms of the time elapsed between the signal triggering tilt of the platform and the onset of EMG activity. This was done separately for each of eight corresponding runs by visual identification of the onset of EMG activity and marking on the graphics terminal of the computer. In order to avoid a bias all latency measurements were done independently by a technician who was not familiar with the expected results.

\section{Patients}

We examined 41 patients in whom it was possible to define from morphological (CT, surgery) or clinical data the localisation of the cerebellar disturbance. The patients were classified into one of the following five groups:

(a) eight patients with Friedreich's ataxia were considered to be representative for a disorder affecting mainly the afferent input to the cerebellum, mediated by the spinocerebellar tracts. In four of them the disease was definitely inherited. According to clinical testing the dysmetria was invariably more prominent in the legs than in the arms. Signs of involvement of the posterior columns (loss of vibration sense and position sense) were observed in all of them. Computed tomography showed intact cerebellar structures without atrophy in all of these patients.

(b) Six patients had an isolated lesion of the lower cerebellar vermis, the vestibulo-cerebellum. Two of them had a tumour (medulloblastoma, astrocytoma), two had bleeding from an angioblastoma. One patient had aplasia of the vermis. In all of them the vermal lesion was verified by CT and surgery. The anterior lobe and the hemispheres of the cerebellum were little or not at all affected. The remaining patient suffered from Hallgreen syndrome (retinitis pigmentosa, deafness, vestibulo-cerebellar ataxia). Neurological examination predominantly showed ataxia of sitting, gait, and stance, gaze nystagmus, and a disturbance of suppression of the vestibulo-ocular reflex by fixation and of pursuit.

(c) Eleven patients suffered from a late cortical atrophy of the anterior lobe, a disease which can be observed in chronic alcoholics with a nutritional deficiency. These patients mainly showed a more or less pronounced ataxia of gait more than stance, with a high frequency tremor while standing and ataxia in the legs, but invariably less in the arms. Polyneuropathy was either absent or moderate. Computed tomography of the 10 men and one woman showed generalised atrophy of the brain to a varying degree with predominance in the vermal and anterior lobe region of the cerebellum.

(d) Seven patients suffered from diseases, which were mainly localised in the cerebellar hemispheres. Three of them had bilateral ischaemic lesions. In the remaining four who exhibited ataxia and dysmetria predominantly of the arms and less of the legs, the cause of the cerebellar disease was unknown.

(e) Nine patients had a cerebellar disease, which according to clinical testing and CT affected all parts of the cerebellum. Three of them suffered from chronic intoxication (two bromide, one diphenylhydantoin), five suffered from a heredoataxia (Gerstman-Sträussler (1), familial spastic ataxia (2), olivo-cerebellar degeneration of Holmes (2)); in one patient the cause of the cerebellar affection could not be classified.

The results of posturography on the stable platform from these patients are reported elsewhere. ${ }^{25}$

For comparison we also investigated a population of 20 healthy subjects matched in age. The comparison of the results of the different patient groups was done by analysis of variance. In a further experiment we performed platform tilts toe-up and toe-down as well, both with open and closed eyes, in 12 patients with atrophy of the anterior lobe and 12 normal subjects. This was done in order to see whether the specific alterations of the EMG response pattern observed after platform tilt toe-up were also present after stretch of the anterior tibial muscle. We also wondered how eye closure, which provokes tremor, alters these EMG responses.

\section{Results}

Mean latencies, standard deviations, and frequencies of occurrence of the three EMG responses of the leg muscles are displayed in table 1. Figure 1 shows examples of averaged rectified EMG responses from representative patients. An analysis of variance showed no significant differences for the latency of the short latency response in the stretched triceps surae muscle. As was expected from the clinical examination the stretch reflex was missing in half of the patients with Friedreich's ataxia. In the remaining four patients the short latency response could be recorded although the tendon reflex could not be obtained by clinical testing. With the parameters of the displacement used for testing, the medium latency response in the triceps surae muscle could be evoked in nearly all normal subjects (Fig 1a) and patients, except for patients with Friedreich's ataxia. For those patients in whom a medium latency response was present the analysis of variance showed a significant difference for the latency of this EMG response which was due to the increase in latency of medium latency (mean difference of + $50.5 \mathrm{~ms}$,) in three patients with Friedreich's ataxia; (multiple comparisons). The late antagonistic response of the anterior tibialis muscle after the platform tilt toe-up could be recorded in all patients and normals. The analysis of variance showed a significant difference of the latency of this response which was again due to patients with Friedreich's 
Table 1 Mean latencies (ms) and standard deviations of the three EMG responses after platform tilt toe-up

\begin{tabular}{|c|c|c|c|c|c|c|c|c|c|c|}
\hline \multirow[t]{2}{*}{ Patient group } & \multirow[t]{2}{*}{$N$} & \multicolumn{3}{|c|}{$M 1$ response $(T S)$} & \multicolumn{3}{|c|}{$M 2$ response $(T S)$} & \multicolumn{3}{|c|}{$M 3$ response $(T A)$} \\
\hline & & $\boldsymbol{A M}$ & $S$ & $N$ & $A M$ & $\boldsymbol{S}$ & $N$ & $\boldsymbol{A M}$ & $S$ & $N$ \\
\hline $\begin{array}{l}\text { Friedreich's ataxia } \\
\text { Vermis } \\
\text { Anterior lobe } \\
\text { Hemisphere } \\
\text { Diffuse } \\
\text { Normals } \\
\text { Sum N } \\
\text { F value } \\
(\text { Fp }<0.05=2.39)\end{array}$ & $\begin{array}{r}8 \\
6 \\
10 \\
6 \\
9 \\
20 \\
59\end{array}$ & $\begin{array}{l}53 \cdot 2 \\
58.0 \\
53.0 \\
50.5 \\
54.5 \\
50.7\end{array}$ & $\begin{array}{r}7.9 \\
14.5 \\
6.6 \\
8.5 \\
10.5 \\
6.8 \\
0.81\end{array}$ & $\begin{array}{r}4 \\
5 \\
10 \\
6 \\
9 \\
20 \\
54\end{array}$ & $\begin{array}{l}154.6 \\
118.0 \\
119.2 \\
102.5 \\
124.9 \\
104.1\end{array}$ & $\begin{array}{r}10.1 \\
10.3 \\
29.9 \\
7.8 \\
29.9 \\
13.5 \\
\\
8.65\end{array}$ & $\begin{array}{r}3 \\
5 \\
9 \\
4 \\
7 \\
18 \\
46\end{array}$ & $\begin{array}{l}296.9 \\
148.7 \\
135.1 \\
135.1 \\
179.2 \\
131.1\end{array}$ & $\begin{array}{l}71 \cdot 7 \\
47 \cdot 1 \\
38 \cdot 3 \\
21 \cdot 8 \\
51 \cdot 6 \\
32 \cdot 0 \\
\\
18 \cdot 42\end{array}$ & $\begin{array}{r}8 \\
6 \\
10 \\
6 \\
9 \\
20 \\
59\end{array}$ \\
\hline
\end{tabular}

M1 = short latency. M2 = medium latency, M3 = long latency.

$\mathbf{A M}=$ arithmetic mean. $\mathbf{S}=$ standard deviation. $\mathbf{N}=$ no of EMG responses.

ataxia (multiple comparisons;). The increase in latency of the long latency response in the anterior tibial muscle by about $170 \mathrm{~ms}$ theoretically could be due to the loss of this long latency "reflex", but the small intraindividual standard deviation favours the assumption of an actual increase in latency. Some of the patients with diffuse cerebellar diseases also showed an increased latency of the long latency response (fig 1e). All of them had clinical signs of an involvement of the spinal proprioceptive pathways.
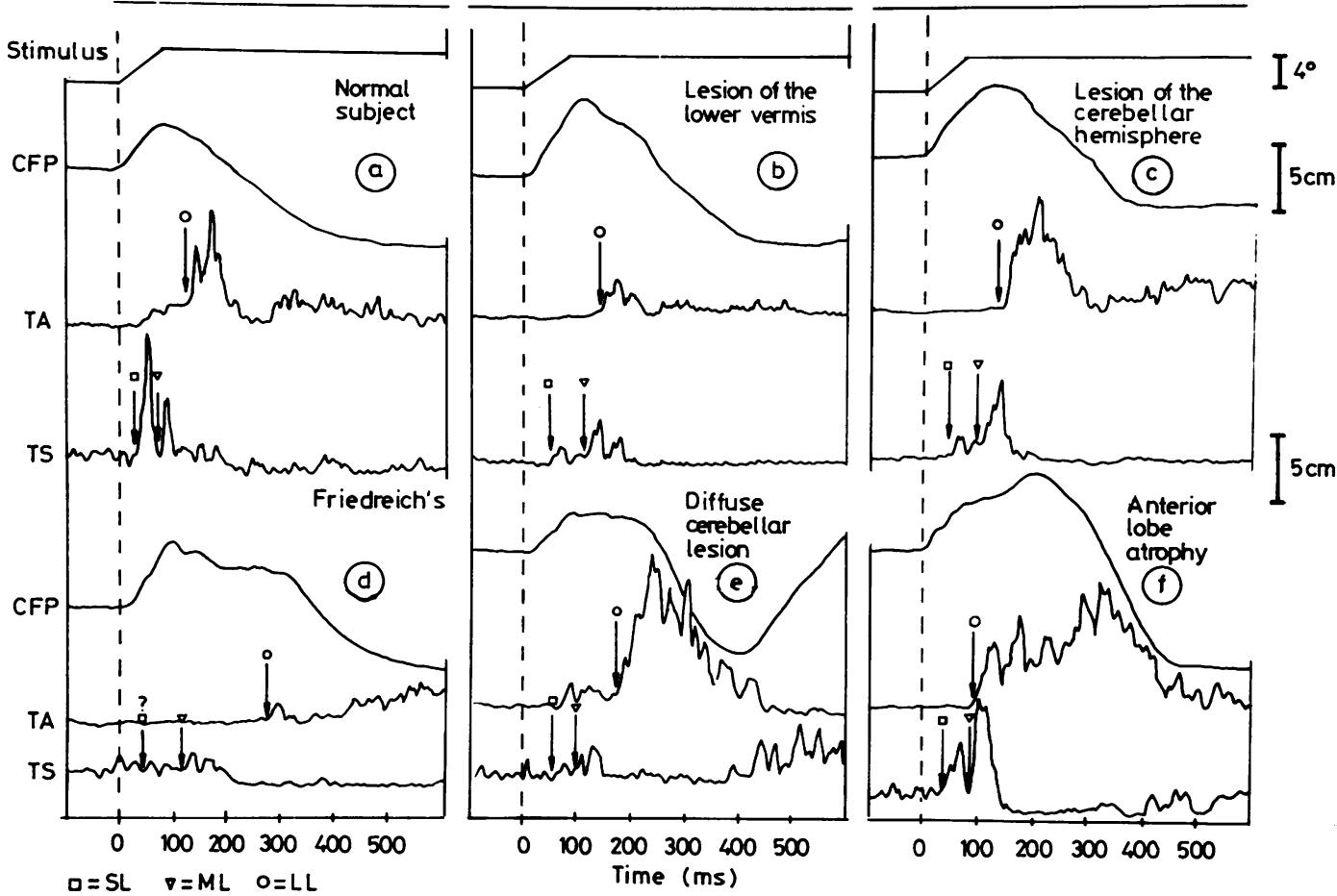

Fig 1 Original averaged EMG recordings from the anterior tibial muscle (TA) and the triceps surae muscle (TS) after platform tilt toe-up $\left(50^{\circ} / \mathrm{s}, 4^{\circ}\right)$. The short latency response is indicated by a square, the medium latency response by a triangle, and the long latency response by a circle. Note the normal latencies of all 3 EMG responses in the normal subject, and with lesions of the lower vermis, lesion of the cerebellar hemisphere and anterior lobe atrophy. Medium latency response is increased in patients with Friedreich's disease, long latency increased in the same patient and in the patient with the diffuse cerebellar lesion. The mechanical consequence of the increased latency of long latency is seen in the recording of the CFP in the patients with Friedreich's disease. The increased amount of long latency is reflected in the plateau of the centre of foot pressure. 
Table 2 Duration of the late antagonistic EMG response after platform tilt (ms). Data from 12 normals 、 and 12 patients with atrophy of the anterior lobe of the cerebellum

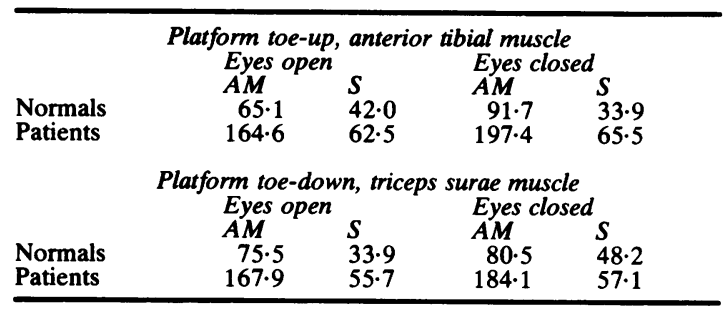

$\mathbf{A M}=$ mean. $\mathbf{S}=$ standard deviation.

The comparison of the long latency in the anterior tibial muscle after platform tilt toe-up and of the triceps surae muscle after tilt toe-down showed no significant difference between 12 normals and 12 patients with atrophy of the anterior lobe: toe-up (tibialis anterior): $131 \cdot 1 \pm 32 \mathrm{~ms}$ in normals and $123.0 \pm 32.5 \mathrm{~ms}$ in patients; toe-down (tripceps surae): $141 \cdot 1 \pm 31.3 \mathrm{~ms}$ in normals and $168.0 \pm$ $46 \mathrm{~ms}$ in patients). Latencies were also independent of whether or not the eyes were open or closed. In contrast to these results, the duration of the antagonistic responses was about 2-3 time larger in patients with atrophy of the anterior lobe (table 2, fig 2). Both populations showed a further increase in duration of the functionally stabilising response when the visual input was missing (eyes closed). The amplitude of the long latency response as determined by the integrated EMG activity was significantly larger in patients with anterior lobe atrophy (normals 40.2 arbitrary units, patients 63.3 ) and again showed a further increase after eye closure (normals 40.9 patients $73 \cdot 3$ ). In contrast to Mauritz et al ${ }^{23}$ who investigated rather advanced cases of the disease we examined a higher proportion of patients with an incipient $3 \mathrm{~Hz}$ tremor of the

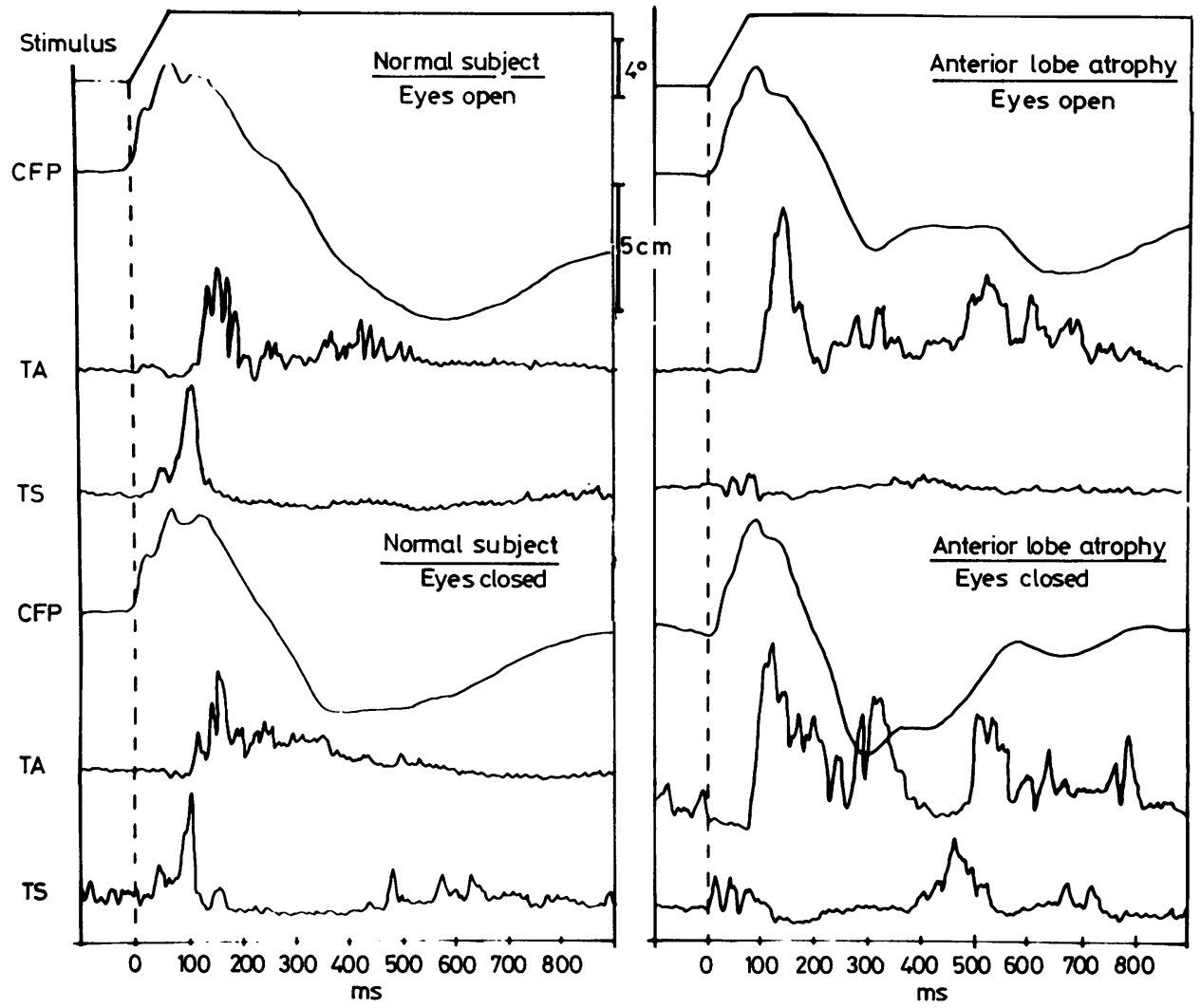

Fig 2 Averaged recordings from the anterior tibial (TA) and the triceps surae (TS) muscles from a normal subject on the left and a patient suffering from atrophy of the anterior lobe of the cerebellum on the right after platform tilt toe-up $\left(50 \%, 4^{\circ}\right)$. Note the increased long latency response of the patient which further increases after eye closure. 


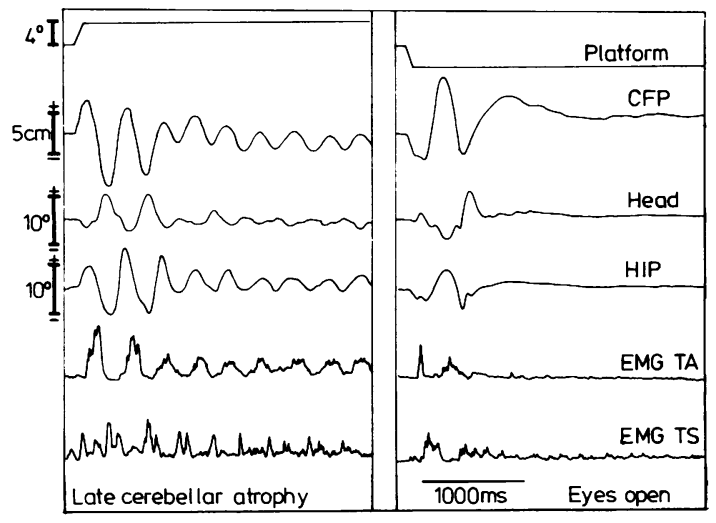

Fig 3 Provocation of the typical $3 \mathrm{~Hz}$ body tremor in a patient with late cerebellar atrophy of the anterior lobe after a platform tilt toe-up (on the left). Note the increased intersegmental stabilisation by counterphase movements of head and hip. The reactions to platform tilt toe-down (on the right) are not different from a normal reaction.

body. Only one patient exhibited this tremor at rest with open eyes. We were able to evoke the truncal tremor by a sudden tilt of the platform toe-up in four patients standing with their eyes open and in eleven of the twelve patients with eyes closed. In some of our patients a directional asymmetry could be observed. In these, tremor was evoked only by platform tilt toe-up, but not by tilt toe-down (fig 3).

\section{Discussion}

Cerebellar dysfunction was investigated in patients in whom the extent and location of the lesion was determined by computed tomography and clinical testing. Four of the five groups examined in this study represent lesions of the different cerebellar subunits potentially involved in stabilisation of posture.

The latency of the stretch reflex in the triceps surae muscle was unchanged in most of our patients. This conforms with the classical assumption of a segmental wiring of this short latency response. The only exception are patients with Friedreich's ataxia in whom peripheral neuropathy and demyelination of the most proximal segment of the afferent fibres is known to occur. Late cortical cerebellar atrophy frequently occurs without a major peripheral neuropathy (60\% in our material).

The medium latency response in the stretched triceps surae muscle corresponds to the functional stretch reflex,,$^{12}{ }^{13}$ the postmyotatic reaction, ${ }^{26}$ and to the $\mathrm{M} 2$ response in arm muscles. ${ }^{11} 27$ In contrast to the results in arm muscles, where cerebellar lesions resulted in a loss or diminuation of the initial com- ponent of $\mathrm{M} 2$ with a delay of $\mathrm{M} 3{ }^{28}$ the medium latency response in leg muscles remains unchanged in latency as long as the dorsal columns and spinocerebellar tracts are not involved (fig 1c, e, f). The considerable delay of the medium latency response in three patients with Friedreich's ataxia cannot be explained by a peripheral delay since all of the three had a normal latency of the stretch reflex and a maximal motor nerve conduction velocity at the lower end of normality. The considerable delay favours a suprasegmental pathway of the "reflex arc" with its afferent inputs mediated by the posterior columns and/or the spinocerebellar pathways. For the ones in whom the medium latency response was missing it cannot be decided where the block was localised.

We have never observed an adaptation of the medium latency response in more than 300 normals and patients recorded so far. This does not disprove the finding of Nashner and Grimm, ${ }^{29}$ who observed a loss of adaptation of the functional stretch reflex in cerebellar patients when they changed the platform movement from a horizontal backward motion to an upward rotation, both stretching triceps surae muscle. We were unable to change in a similar way the experimental paradigm.

In contrast to the medium latency response, the antagonistic long latency response in the anterior tibial muscle could be evoked in all normals and patients. The latency of this response was unchanged in all patients suffering from an isolated lesion of the cerebellum. Apparently the cerebellum is not involved in the exact timing of this postural response. Patients with Friedreich's ataxia mainly suffering from a degeneration of afferent spinocerebellar pathways had a massive increase in latency of the first EMG response in this muscle. The latency of the long latency response was also delayed in those patients with diffuse cerebellar lesions who additionally had clinical symptoms pointing towards an involvement of the posterior columns. Whether the long latency response is only delayed or missing and replaced by a voluntary muscle contraction cannot definitely be decided at present. The relatively small intraindividual variation (much larger with voluntary movement ${ }^{3}$ ), the lack of a training effect in repeated trials, and the correlation of the increase in latency with the severity of the disease favour the assumption of a delayed long latency response. Since none of the patients exhibiting a lesion confined to the cerebellum alone shows a delay of the long latency response, one may be tempted to conclude that the initiation of this response is independent of the cerebellum. Its duration and amplitude, however, as evidenced by the group with cortical anterior lobe atrophy seems to 
depend on spinocerebellar activity.

Patients suffering from atrophy of the anterior lobe of the cerebellum had normal latencies of short, medium and long latency responses. The earlier explanation for the generation of the $3 \mathrm{~Hz}$ body tremor given by Mauritz et al. ${ }^{23}$ and Dichgans and Maurit $^{24}$ therefore requires modification. These authors stimulated both tibial nerves in the popliteal fossa. The discharge of the anterior tibial muscle with a latency of $120 \mathrm{~ms}$ after the stimulus was considered to be a stretch reflex of this muscle, elicited by the contraction of the triceps surae and the resulting backward sway of the body. Synchronisation of stretch reflexes, delayed and enhanced long loop responses were supposed to sustain the tremor. We were never able to evoke the stretch reflex in the anterior tibial muscle by means of a plantar flection of the foot by tilting the platform toe-down. ${ }^{3}$ The $t i b$ 1 response of Mauritz et $a^{23}$ obviously corresponds to our stabilising long latency response. We now explain the $3 \mathrm{~Hz}$ tremor by the increased duration and amplitude of the long latency response in the functionally stabilising antagonist. This increased activity leads to an overshoot of the correction, resulting in a forward sway of the body which in turn is compensated by activity of similar duration and amplitude in the triceps surae muscle and so on.

Some patients with lesions of the lower vermis or the cerebellar hemispheres also exhibited an increased duration of the long latency response. The long latency response was (in contrast to the patients with atrophy of the anterior lobe) tonically increased whereas in the alcoholics long latency was phasic.

The long latency response of the anterior tibial muscle is not equivalent to the "shortening reaction" after passive shortening of the muscle in sitting of supine subjects. ${ }^{30}$ The shortening reaction can be evoked in $56 \%$ of normals in the anterior tibial muscle and in $6 \%$ in the triceps surae muscle,$^{30}$ whereas the long latency response can be seen in $100 \%$ of normals and patients in both muscles when standing. The shortening reaction is known to be exaggerated in patients with Parkinson's disease while the long latency response was normal in our study of 36 patients with Parkinson's disease (unpublished).

This work was supported by the Deutsche Forschungsgemeinschaft (DI 278/1-2).

\section{References}

' Allum JHJ, Büdingen JH. Coupled stretch reflexes in ankle muscles: An evaluation of the contribution of active muscle mechanisms to human postural stability. Progr Brain Res 1979;50:185-95.
${ }^{2}$ Diener HC, Bootz F, Dichgans J, Bruzek W. Variability of postural "reflexes" in humans. Exp Brain Res 1983;52:423-8.

${ }^{3}$ Diener HC, Dichgans J, Bootz F, Bacher M. Early stabilization of human posture after a sudden disturbance: Influence of rate and amplitude of stretch. Exp Brain Res 1984, in press.

4 Allum JHJ, Mauritz KH, Vögele H. Stiffness regulation provided by short-latency reflexes in human triceps surae muscles. Brain Res 1982;234:159-64.

5 Allum JHJ, Mauritz KH, Vögele $\mathrm{H}$. The mechanical effectiveness of short latency reflexes in human triceps surae muscles revealed by ischemia and vibration. Exp Brain Res 1982;48:153-591.

- Elner AM, Popov KE, Gurfinkel VS. Changes in stretch reflex system concerned with the control of postural activity of human muscle. Agressologie 1972; 13D:19-20.

${ }^{7}$ Gottlieb GL, Agarwal GC. Response to sudden torques about ankle in man. I. Myotic reflex. J Neurophysiol 1979;42:91-106.

${ }^{8}$ Chan CWY, Melville-Jones G, Kearney RE, Watt DGD. The "late" electromyographic responses to limb displacement in man. I. Evidence for supraspinal contribution. Electroencephalogr Clin Neurophysiol 1979;46:173-81.

${ }^{9}$ Lee RG, Tatton GW. Motor responses to sudden limb displacement in primates with specific CNS lesions and in human patients with motor system disorders. Can J Neurol Sci 1975;2:285-93.

${ }^{10}$ Hammond PH. An experimental study of servo action in human muscular control. In: Proc III International Conference in Medical Electronics. London. Inst. Electr. Eng. p 190-9.

"Marsden CD, Merton PA, Morton HB. The sensory mechanism of servo action in human muscle. $J$ Physiol (London) 1977;265:521-35.

${ }_{12}$ Melville Jones G, Watt DGD. Observations on the control of stepping and hopping movements in man. $J$ Physiol (London) 1971;219:709-27.

${ }^{13}$ Nashner LM. Adapting reflexes controlling the human posture. Exp Brain Res 1976;26:59-72.

14 Iles JF. Response in human pretibial muscles to sudden stretch and nerve stimulation. Exp Brain Res 1977;30:451-70.

15 Phillips CG. Motor apparatus of the baboon's hand. Proc Roy Soc 1969;173:141-74.

${ }^{16}$ Ghez C, Shinoda Y. Spinal mechanisms of the functional stretch reflex. Exp Brain Res 1978;32:55-68.

${ }_{17}$ Miller AD, Brooks VB. Late muscular responses to arm perturbations persist during supraspinal dysfunction in monkeys. Exp Brain Res 1981;41:146-58.

${ }^{18}$ Hagbarth KE, Hägglund JV, Wallin EU, Young RR. Grouped spindle and electromyographic response to abrupt wrist extension movements in man. J Physiol (London) 1981;312:81-96.

${ }^{19}$ Diener HC, Dichgans J, Bootz F. Functional plasticity of spinal and supraspinal reflexes in maintaining upright stance. Adv Oto-Rhino-Laryngol 1983;30:288-90.

20 Thomas A. Atrophie lamellaire des cellules de Purkinje. Rev Neurol (Paris) 1905;13:917-24.

${ }^{21}$ Marie P, Foix C, Alajouanine T. De l'atrophie cerebel- 
leuse tardive à prédominance corticale (atrophie parenchymateuse primitive des lamelles du cervelet, atrophie paleocerebelleuse primitive). Rev Neurol (Paris) 1922;29:849-85;1082-111.

${ }^{22}$ Victor M, Adams RD, Mancall EL. A restricted form of cerebellar cortical degeneration occurring in alcoholic patients. Arch Neurol 1959;1:579-688.

${ }^{23}$ Mauritz KH, Schmitt C, Dichgans J. Delayed and enhanced long latency reflexes as the possible cause of postural tremor in late cerebellar atrophy. Brain 1981;104:97-116.

${ }^{24}$ Dichgans J, Mauritz KH. The pathophysiology of postural imbalance in cerebellar patients. In: Honrubia V, Brazier MA, eds. Nystagmus and Vertigo: Clinical Approaches to the Patient with Dizziness. New York: Academic Press 1982:179-89.

${ }^{25}$ Diener HC, Dichgans J, Bacher M, Gompf $B$. Quantification of postural sway in normals and patients with cerebellar diseases. Electroencephalogr Clin Neurophysiol 1983;57:134-42.
${ }^{26}$ Gottlieb GL, Agarwal GC. Response to sudden torques about ankle in man. II. Postmyotic reactions. $J$ Neurophysiol 1980;43:86-101.

${ }^{27}$ Lee RG, Tatton WG. Long latency reflexes to imposed displacements of the human wrist: Dependence on duration of movement. Exp Brain Res 1982;45:20716.

${ }^{28}$ Marsden CD, Merton PA, Morton HB, Hallett M, Adam J, Rushton DN. Disorders of movement in cerebellar disease in man. In: Rose F, ed. Physiological Aspects of Clinical Neurology. Oxford: Blackwell Sciences Publications 1977:179-99.

${ }^{29}$ Nashner LM, Grimm RJ. Analysis of multi loop dyscontroles in standing cerebellar patients. In: Desmedt JE, ed. Cerebral Motor Control in Man: Long Loop Mechanisms. Basel: Karger 1978:300-19.

${ }^{30}$ Katz R, Rondot P. Reaction to passive muscle shortening in normal man. Electroencephalogr Clin Neurophysiol 1978;45:90-99. 